Electronic version of an article published as [European Journal of Operational Research, 2008, Vol. 184, No. 1, p. 1-12]

[DOI: http://dx.doi.org/10.1016/j.ejor.2006.10.028] @ [Elsevier] 


\title{
Selecting and adapting weekly work schedules with working time accounts: A case of a retail clothing chain"
}

\author{
Rafael Pastor $^{a, b}$ and Jordi Olivella ${ }^{b}$ \\ ${ }^{a}$ IOC Research Institute, Technical University of Catalonia, Barcelona, Spain \\ ${ }^{\mathrm{b}}$ Department of Management, Technical University of Catalonia, Barcelona, Spain
}

\begin{abstract}
A case of selection and adaptation of weekly work schedules is presented. Weekly work schedules in two franchises of an important retail clothing chain have to be established. Working time accounts are used: each week, an employee can owe the company a certain number of hours or vice versa. Nevertheless, over a certain threshold, the hours have to be paid for by the company and the account balance returns to zero. A minimum and desired level of capacity of employees is contemplated. Hierarchically, the planned capacity must attempt to reach the minimum level; then it must fit a desired level as much as possible. At present, the task of allocation and the final adjustment of schedules is done manually, which is difficult, ineffective and often inaccurate. The procedure proposed is divided into two phases. Firstly, a work schedule, selected from a list, is assigned to each worker; a mixed linear program, followed by a local optimization process, is used. In the second phase, the work schedules are modified according to predefined rules: if there is a surplus of capacity, work schedules are reduced, and if there is a shortage, work schedules are extended. The company considers the results to be satisfactory.
\end{abstract}

Keywords: OR in manpower planning; Workforce planning; Work scheduling; Case study

"Supported by the Spanish MCyT Project DPI2004-05797 co-financed by the FEDER. 


\section{Introduction}

This paper deals with the problem of selecting and adapting work schedules in a services company, specifically a retail clothing chain. Their policy about workers' schedules used to take a complex mix of restrictions into account. The process of allocating and modifying schedules was timeconsuming and unreliable. Our goal was to make this work better and easier while maintaining all aspects that the company wanted to consider.

The problem has two unusual characteristics: on the one hand, the working time accounts concept is used; on the other hand, the employee capacity must be planned to reach the desired level as much as possible, while attempting to guarantee minimum levels of capacity at all periods within the planning horizon.

The company studied is a retail chain that sells clothing. It began its activities in the 1950s; it currently has around 800 establishments in 15 countries, more than 30,000 employees and over 1500 million euros in annual sales.

The shops are open every week of the year and a work schedule has to be assigned to each employee for each week he or she has to work. There are two hierarchical objectives. First, an attempt to guarantee minimum capacity levels must be made. Second, the planned capacity must fit a desired level as much as possible. It is necessary for each available employee to have a different weekly work schedule each week for two reasons. Firstly, the employees who are available may change from one week to another (due to holidays, training, hiring and illness). Secondly, the level of required capacity changes every week because it is generated on a weekly basis according to the sales records for the same week of the previous year. The shops have between 25 and 50 workers, although there are also bigger ones. They plan the work schedules for their sales departments, which sell men's, women's or children's clothing, with a certain number of workers per department within the same bounds.

The establishment of the required capacity levels is beyond the scope of this study. However, it must be mentioned in order to describe the case to be solved in detail. The sales goal for the week being planned, known as the budget $(\Psi)$, is calculated by multiplying the sales records for the same week of the previous year by a certain number higher than 1 . Moreover, particular characteristics of the week, such as public holidays or campaigns, are taken into account. The ratio $\mu$ is the sales goal per hour of work. The total number of hours of capacity required for the week is therefore $\Psi / \mu$. Finally, each shop has a required capacity pattern that corresponds to a baseline budget $\Psi^{0}$. This pattern is obtained from the historical data on work time schedules established by the manager of the shop according to the corresponding budgets. The required capacity level is obtained based on the required capacity pattern and the relationship between $\Psi$ and $\Psi^{0}$. The manager of the shop should obviously make final adaptations based on public holidays, special promotions and so on.

The problem has two main characteristics. Firstly, the number of work hours assigned to each worker each week can be more or less than the usual workweek, within the bounds agreed on by the company and the workers. For example, schedules can change from $22 \mathrm{~h}$ to $26 \mathrm{~h}$ of work for a worker who is contracted to work $24 \mathrm{~h}$ a week. In such cases, a working time account is used. It is possible that the worker owes working hours to the company, in which case the worker will have to work longer weekly schedules at a later date (negative balance). More commonly, the company owes working hours to the worker, who will be compensated by shorter weekly schedules in the future (positive balance). Nevertheless, over a certain threshold, the hours have to be paid for by 
the company: the positive balance is paid, at the normal rate, and the account balance returns to zero. Then, initial balance of each worker's working time account is known when a week is planned. Working time accounts allow great flexibility, because adjusting schedules to the required capacity levels becomes possible. Planning, however, is more complicated.

The company is subject to the sector's collective agreement, which allows the flexibility of schedules, although to a limited extent. A complementary agreement between shops and workers defines the next two specific characteristics. On the one hand, there is a set of possible contracts (of $40,30,24$ or $12 \mathrm{~h} /$ week) and a list of possible weekly work schedules (here called standard) for each of them (for example, 50 standard schedules for contracts of $40 \mathrm{~h} / \mathrm{week}$ ). These standard schedules adhere to the work hours in the contract. On the other hand, a set of rules that allows the duration of the standard schedules assigned to workers to be extended or reduced is also accepted (except for contracts of $40 \mathrm{~h} /$ week).

A second main characteristic of the problem is that the planned capacity, that is the results of the weekly schedules chosen, must fit as much as possible a desired level of capacity. In either case, a capacity equal to or above an established minimum level should be guaranteed for any period in the planning horizon. It is considered that without this minimum capacity, it would not be possible to carry out certain necessary tasks (for example, at least one employee is necessary in each section or on each floor to attend to the public, to serve at the cash register and to prevent shoplifting).

At present, the task of allocating and modifying schedules is done manually by the manager of the shop. On paper, using different colored pencils, he or she assigns a standard schedule to each available employee, and, if necessary, changes the schedules according to the established rules. Other changes (absences, delays, unplanned increases or decreases in working hours, etc.) are registered using the same medium. As mentioned above, working time accounts complicate system management. All data has to be transmitted to a central administrative system, not only in exceptional circumstances such as absence, but also for the payment of positive balances when they reach the threshold that must be reported. Doing this task manually is laborious (the manager spends half a morning on it); it can be improved (now it is ineffective); mistakes are frequent, both in the interpretation and in time account records (due to the paper format); and the result depends on the experience, intuition or even the mood of the planner.

A procedure for defining the schedule of each worker for a certain week is proposed. The method assigns a standard work schedule to each available worker and then, according to certain rules, it modifies the assigned schedule by attempting to guarantee minimum capacity levels for each period (depending on the shop, an hour or half-hour) in the planning horizon (a week). A secondary objective is to fit, as much as possible, planned capacity to the desired level. The procedure is divided into two phases. In the first phase, a standard schedule is assigned to each worker who is available for work that week. Initially, a mixed integer linear program that assigns a standard schedule to each worker is solved, in a limited calculation time; then, if an optimal result cannot be guaranteed, a local optimization algorithm is applied. In the second phase, the standard schedules that have been assigned are modified: initially, hours of work are eliminated (or re-assigned) in periods in which the desired capacity is exceeded; finally, schedules are extended, if this is necessary and possible, to cover shortages.

Planning is carried out for a set of consecutive weeks. The weeks are planned sequentially in accordance with the designed procedure. The initial balance of each worker's working time account is known. In the first phase of the procedure, a standard schedule that maintains the number of 
weekly contractual hours is always assigned (i.e., working time accounts are not used). In the second phase, the standard schedules are adapted by using working time accounts: if there is a surplus of capacity, work hours are reduced (for the workers whose balance is most favorable to the company among those for whom the reduction is feasible); and if there is a shortage of capacity, work hours are increased (for the workers whose balance is most unfavorable to the company among those for whom the reduction is feasible).

The rest of the paper is organized as follows: Section 2 provides a review of the literature. The procedure for selecting and adapting weekly schedules with working time accounts is presented in Section 3. Section 4 describes the computational experiment that was carried out to validate the procedure, its results and the improvements obtained by using the method. Finally, Section 5 is devoted to the conclusions.

\section{Literature review}

The assigning of schedules to workers, which is a classic topic in work time organization literature, generates different types of problems (Blochliger, 2004). Corominas and Crespán (1993) proposed the following classification: (i) staff dimensioning with prearranged work schedules (Dantzig, 1954); (ii) individual assignment of prearranged work schedules (Hung, 1997); (iii) assignment of implicitly defined work schedules (Day and Ryan, 1997); and (iv) determination of schedules according to certain conditions - for example, scheduling rotation shifts, as in Laporte (1999). This application considers two of these problems: firstly, the individual assignment of prearranged work schedules, and, secondly, the determination of schedules when initially considered schedules are modified.

In service companies, storage is not possible and capacity is directly proportional to the number of workers. Therefore, improving the allocation of work schedules is the least costly way (and sometimes the only way) of dealing with variable demand and improving competitiveness (Oke, 2000). Retailing has specific characteristics, such as opening hours that far exceed the daily working hours of an employee, and the client's presence depends on the time and changes from one day to another. These aspects determine the scheduling problem to be solved (Sudden and Johns, 1995). Even though retailing generates complex work scheduling problems (Ernst et al., 2004), the literature pays them scant attention, Lam et al. (1998) being a major exception.

According to Thompson (1997a), work schedule assignment problems can be easily formulated as mathematical programs, although it is very difficult to solve them optimally as quickly as companies would like (sometimes in a few minutes). Consequently, heuristics (McGinnis et al., 1978), meta-heuristics (Thompson, 1996) and simulation (Freemason et al., 1998) have usually been applied.

From the earliest literature on work time scheduling (Dantzig, 1954), the goal of most papers dealing with the design or management of work time scheduling systems has been to guarantee minimum capacity. More recently, a desired capacity level has been considered: Thompson (1997b) states that the distinction between desired and minimum service level is new in work time scheduling literature. According to him, papers about methods that guarantee minimum capacity and whose secondary objective is to fit a desired capacity level are scarce. Adjusting measurements to the desired level admits several approaches: to consider shortages or shortages and surpluses, and linear (Keith, 1979) or non-linear (Corominas et al., 2005) functions. 
As stated above, having a list of possible work time schedules for each type of contract is a widespread practice, but the authors have not found the use of the strategy of modifying the initial assignment in the literature.

The use of working time accounts makes obtaining favorable schedules easier (Pinker and Larson, 2003), since it allows work to be assigned over or below the number of contract hours. Differences are accumulated in the working time accounts and compensated for later. This scheme has existed since the 1980s (Berg et al., 2004) and it is increasingly widespread in Europe, as reported by Lehndorff (1999).

\section{A procedure for selecting and adapting weekly work schedules}

\subsection{Initial considerations}

The procedure designed begins by assigning a standard schedule to every worker. The work schedules are then modified according to a set of rules. The main objective is to attempt to guarantee a minimum capacity level for each period in the planning horizon, and the secondary objective is to fit a desired capacity level. The procedure is composed of two phases with two subphases each (see Fig. 1). In Phase 1, a standard work schedule is assigned to each available worker: firstly, in Sub-phase 1.1, a standard schedule is assigned to each worker by solving, within a maximum calculation time, a mixed integer linear program (MILP); if an optimal solution cannot be guaranteed, a local optimization algorithm is applied in Sub-phase 1.2. Phase 2 improves the solution by modifying the assigned standard schedules: firstly, Sub-phase 2.1 eliminates or reassigns work hours in periods in which the desired capacity level is exceeded; and, secondly, in Sub-phase 2.2 schedules are extended to cover shortages, when the rules limiting changes in the work schedule allow this.

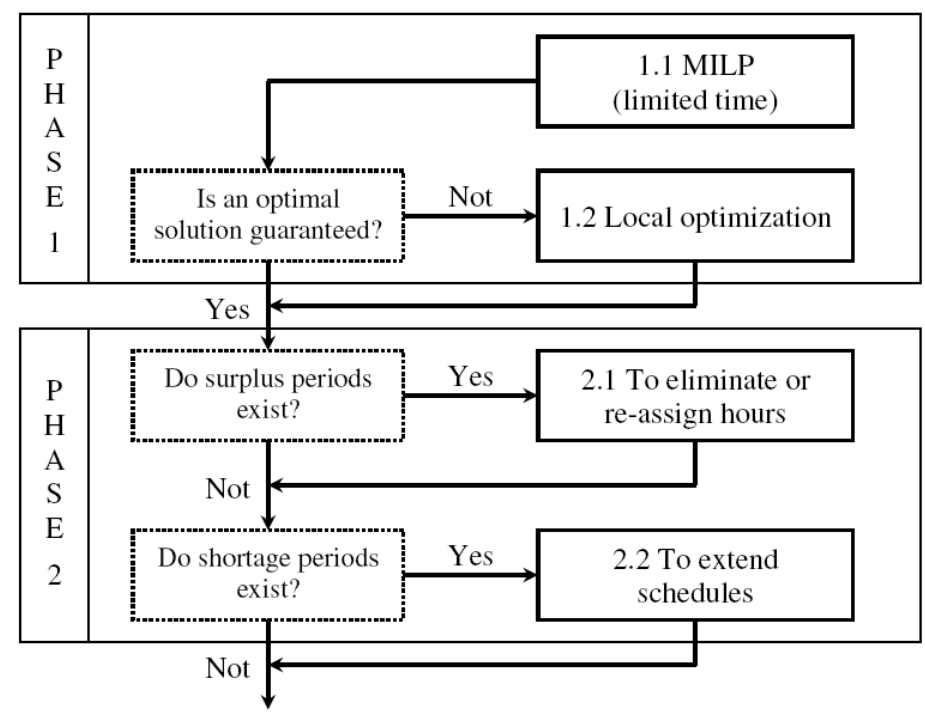

Fig. 1. Phases and sub-phases of the procedure designed.

To plan a workweek, the data that are provided by the company and are used in the decision process are the following: 
(a) Planning horizon and periods: basic unit of planning (depending on the shop, an hour or halfhour).

(b) Set of available employees and, for each of them, their type of contract (40, 30, 24 or $12 \mathrm{~h} /$ week) and the number of hours of work that each worker owes the company or the company owes him/her (negative or positive balance, respectively).

(c) Minimum and desired capacity for each period in the planning horizon.

(d) List of possible standard schedules for each type of contract.

(e) Set of rules regulating the changes allowed in standard schedules.

The procedure attempts to guarantee the minimum capacity levels, and fits, as far as is possible, the desired capacity level, taking shortages and surpluses into consideration. To achieve the two goals, the evaluation function proposed by Corominas et al. (2005) was adapted. In Corominas et al. (2005), workforce capacity has to be fitted to the different types of tasks to be executed; here it is fitted to the desired capacity in each period. Thus, the function can be adapted directly.

The evaluation function by Corominas et al. (2005) has a term for shortage, $\Phi\left(D_{t}, D M_{t}, \delta_{t}^{-}\right)$, and a term for surplus, $\Omega\left(D_{t}, \delta_{t}^{+}\right)$, in relation to the desired value. For period $t, D M_{t}$ and $D_{t}$ are the minimum and desired capacity levels, respectively and $\delta_{t}^{-}$and $\delta_{t}^{+}$are the shortage and surplus, respectively. The function $\Phi\left(D_{t}, D M_{t}, \delta_{t}^{-}\right)$is a multipurpose non-linear convex function. It seems logical that shortages have a non-linear effect, although being convex, and then relative shortages are used; it is clear that a 1 unit shortage is not the same with a desired capacity of $2(50 \%)$ than with a desired capacity of 10 (only $10 \%$ ). Therefore, function $\Phi$ minimizes the relative shortages and distributes them homogeneously among the different periods of time (it is assumed that a solution in which the shortages are regularly distributed among the periods is preferred to another in which the total shortage accumulates exclusively in one or a few time periods only). Moreover, function $\Phi$ avoids, if possible, solutions in which the lower bounds on the capacities are not fulfilled in order to try to guarantee the minimum level of service; if this is not possible, shortages with regard to the minimum are severely penalized. Any solution (schedule assignments to workers) is therefore feasible, because an attempt to reach the minimum capacity level is included in the objective function (strongly penalized) instead of including it as a constraint. Similarly, the non-linear convex function $\Omega\left(D_{t}, \delta_{t}^{+}\right)$has been used to minimize relative surpluses and to distribute them homogeneously among the different periods. The exact definitions of functions $\Phi\left(D_{t}, D M_{t}, \delta_{t}^{-}\right)$and $\Omega\left(D_{t}, \delta_{t}^{+}\right)$can be found in Corominas et al. (2005).

\subsection{Phase 1: Assignment of standard work schedules}

Phase 1 consists in assigning one standard work schedule to each worker that is available for work in the week that is being scheduled. A mixed integer linear program (MILP) is solved within a maximum calculation time and, if the optimality of the solution cannot be guaranteed, a local optimization procedure is applied. 


\subsubsection{Sub-phase 1.1: MILP model}

A MILP assigns standard work time schedules to all available workers. The main objective is to attempt to guarantee a minimum capacity for each period in the planning horizon, and the secondary objective is to fit a desired level of capacity. As stated above, this fitting implies minimizing the relative shortages and surpluses and distributing them homogeneously among the different periods of time.

\section{Data}

$T$

Number of periods in the planning horizon

$W$

Number of staff members

$D_{t}$

Desired value of the number of workers assigned to period $t(t=1, \ldots, T)$

$D M_{t}$

Minimum number of workers assigned to period $t(t=1, \ldots, T)$

$L_{i}$

Set of possible standard schedules for worker $i(i=1, \ldots, W)$. Schedules of set $L_{i}$ are defined by means of a matrix of binary elements, meaning that if $a_{j t}=1$ worker $i$ following schedule $j$ has to work in period $t\left(j \in L_{i} ; t=1, \ldots, T\right)$

$\alpha, \beta$

Parameters for weighing the different parts of the objective function $(\alpha+\beta=1)$

\section{Variables}

$X_{i j}$

Binary variable, which is 1 if schedule $j$ is assigned to worker $i\left(i=1, \ldots, W ; j \in L_{i}\right)$

$\delta_{t}^{-}$

Shortage with regard to desired capacity for period $t(t=1, \ldots, T)$

$\delta_{t}^{+}$

Surplus with regard to desired capacity for period $t(t=1, \ldots, T)$

Model 


$$
\begin{aligned}
& {[\mathrm{MIN}] Z=\alpha \cdot\left[\sum_{t=1}^{T} \Phi\left(D_{t}, D M_{t}, \delta_{t}^{-}\right)\right]+\beta \cdot\left[\sum_{t=1}^{T} \Omega\left(D_{t}, \delta_{t}^{+}\right)\right]} \\
& \sum_{j \in L_{i}} X_{i j}=1 \quad(i=1, \ldots, W)_{(2)}^{(1)} \\
& \sum_{i=1}^{W} \sum_{j \in L_{i}} X_{i j} \cdot a_{j t}+\delta_{t}^{-}-\delta_{t}^{+}=D_{t} \quad(t=1, \ldots, T)_{(3)} \\
& 0 \leq \delta_{t}^{-} \leq D_{t} \quad(t=1, \ldots, T)_{(4)} \\
& X_{i j} \in\{0,1\} \quad\left(i=1, \ldots, W ; j \in L_{i}\right) . \quad \text { (5) }
\end{aligned}
$$

Eq. (1) is the objective function, which includes the penalties associated with the shortage with regard to minimum and desired capacity, and the surplus over the desired capacity. Eq. (2) imposes one and only one schedule to every worker. Eq. (3) forces that, in every period, present employees plus shortage minus surplus is equal to the desired capacity level. Eq. (4) establishes lower and upper bounds to shortage. Finally, Eq. (5) expresses the binary character of variables $X_{i j}$.

Due to the non-linearity of functions $\Phi\left(D_{t}, D M_{t}, \delta_{t}^{-}\right)$and $\Omega\left(D_{t}, \delta_{t}^{+}\right)$, the model described is a non-linear mixed integer mathematical program whose difficult to be solved is well-known. However, as both functions are convex and the objective function separable, their linearization is trivial. As explained in Corominas et al. (2005), when the shortage function $\Phi\left(D_{t}, D M_{t}, \delta_{t}^{-}\right)$is linearized, solutions in which the minimum capacity levels are not fulfilled are avoided, if possible.

\subsubsection{Sub-phase 1.2: Local optimization}

The local optimization algorithm takes an initial incumbent solution and applies an iterative improvement process. The solution provided by Sub-phase 1.1 is taken as the first incumbent solution. At each iteration, a neighborhood of the incumbent solution is generated when schedule $h$ assigned to worker $i$ is replaced by every other schedule $j$, where $j \neq h$ and $j \in L_{\mathrm{i}}$. If the best neighbor solution is better than the incumbent solution, it becomes the new incumbent solution; otherwise, the same process is applied to worker $i+1$. The first worker is selected randomly. When all workers have been considered without any improvement, the process ends. Fig. 2 shows the pseudo-code of the algorithm. 
MILP solution is the first incumbent solution $\bar{X}$ with value $\bar{Z}: \bar{X}=X_{M L P}$ and $\bar{Z}=Z_{M L P}$.

Select randomly a worker $i$.

$\underline{\text { Do }}$

Generate the neighborhood $\{\bar{X}\}_{\text {neig }}$ of the incumbent solution, replacing schedule $h$ assigned to $i$ in

$\bar{X}$ with every other schedule $\mathrm{j}$, where $\mathrm{j} \neq \mathrm{h}$ and $\mathrm{j} \in \mathrm{L}_{\mathrm{i}}$.

If the best neighbor is better than the incumbent solution $\left(\min \{\bar{Z}\}_{\text {neig }}<\bar{Z}\right)$ then

$$
\bar{X}=X_{\text {neig }} \mid Z_{\text {neig }}=\min \{\bar{Z}\}_{\text {neig }} \text { and } \bar{Z}=\min \{\bar{Z}\}_{\text {neig }}
$$

end if

If $i=W$ then

$i=1$

$\underline{\text { else }}$

$i=i+$

end if

until no improvement is made in $W$ iterations

Fig. 2. Pseudo-code of Sub-phase 1.2.

\subsubsection{Analysis of Phase 1 results}

In some cases, it is possible to guarantee in a short calculation time $(120 \mathrm{~s})$ that the solution obtained by the MILP is optimal. When the local optimization sub-phase is necessary, some improvements are also obtained in a brief calculation time (about $60 \mathrm{~s}$ ).

Fig. 3 shows an example of Phase 1 results. The planned capacity with work standard schedules fulfils the minimum capacity requirement and follows the tendencies of the desired capacity level. Nevertheless, in some periods there are differences with the desired capacity, especially at midday - this is due to the overlap between the end of morning shifts, the start of afternoon shifts and meal breaks (for workers who work $40 \mathrm{~h} /$ week).

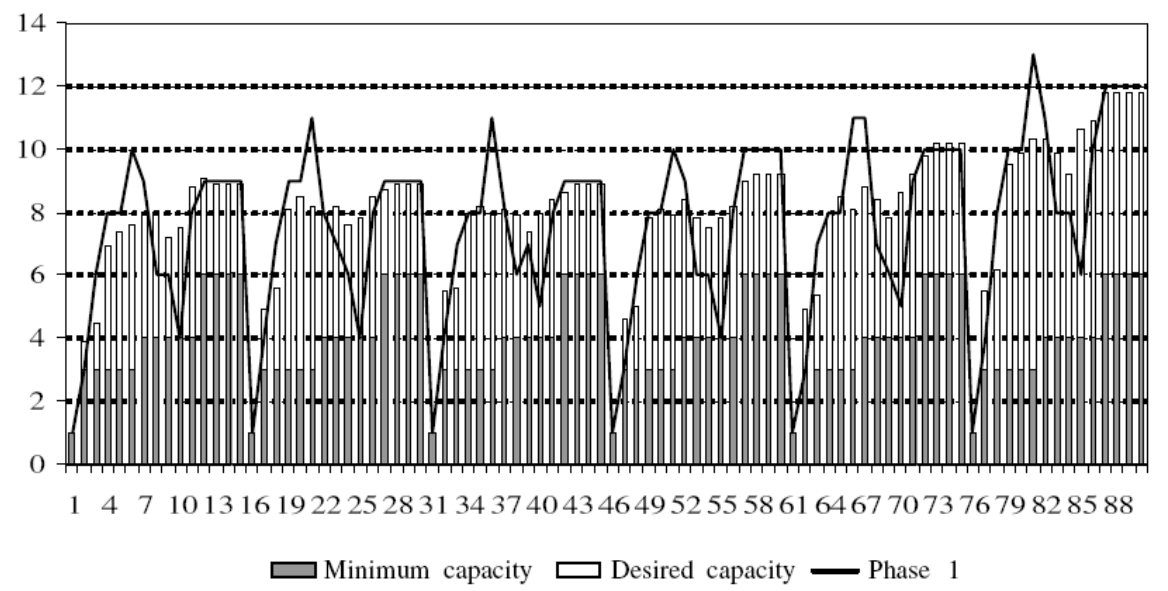

Fig. 3. Example of a Phase 1 solution.

\subsection{Phase 2: Adaptation of standard schedules}

In Phase 2, the standard work schedules assigned (that is, the solution for Phase 1) are modified: firstly, by suppressing or re-assigning work hours to eliminate periods of surplus, if there is a 
surplus of capacity, and then by increasing work hours to cover shortages. All modifications are carried out according to established rules.

\subsubsection{Schedule modification rules}

The rules for modifying standard work schedules, which are established to fit planned capacity to the desired capacity level as far as is possible (and which satisfy, whenever possible, the minimum capacity), are the following:

R1. In periods in which there is a surplus, unnecessary hours are suppressed. These hours are added to periods of shortage, whenever possible; otherwise, they remain as owed by the worker to the company, and are accumulated in the working time accounts.

R2. In the case of shortages, work hours are increased. They are owed by the company to the worker, and are accumulated in the working time accounts.

R3. Rules for eliminating, adding or moving (for eliminating and adding subsequently) working hours:

- $40 \mathrm{~h} /$ week schedules cannot be modified.

- Changes must not cause gaps in the schedules.

- Changes in the number of work hours are limited by worker and/or schedule type: the maximum number of hours/day that can be suppressed/added to the beginning or the end of the schedule assigned; the maximum number of hours that can be suppressed/added per day; and maximum number of hours that can be added per week.

- The number of hours that can be added weekly for the whole workforce is also limited.

\subsubsection{Sub-phase 2.1: Elimination or re-assignment of working hours (surplus case)}

The objective is to suppress, according to the established rules, surpluses in the periods whenever they exist. The solution provided by Phase 1 is taken as the initial incumbent solution. Every period of time $t$ is checked to detect surpluses. Whenever applicable, a set of solutions with one work hour changed from period $t$ to a shortage period is generated; the solutions involving every worker present in period $t$ that fulfills the rules are considered. If these solutions exist, the best of them becomes the incumbent solution; otherwise, the unnecessary work hour is suppressed. This affects the worker present in $t$ with the highest positive balance in his/her work time account, and, in the case of a tie, the worker with the fewest changes in his/her standard schedule is chosen. The work hour that has been eliminated is included in the working time account of the worker affected. The process begins in $t=1$ and ends when all periods in the horizon have been considered without any improvement. Fig. 4 shows the pseudo-code of the procedure. 


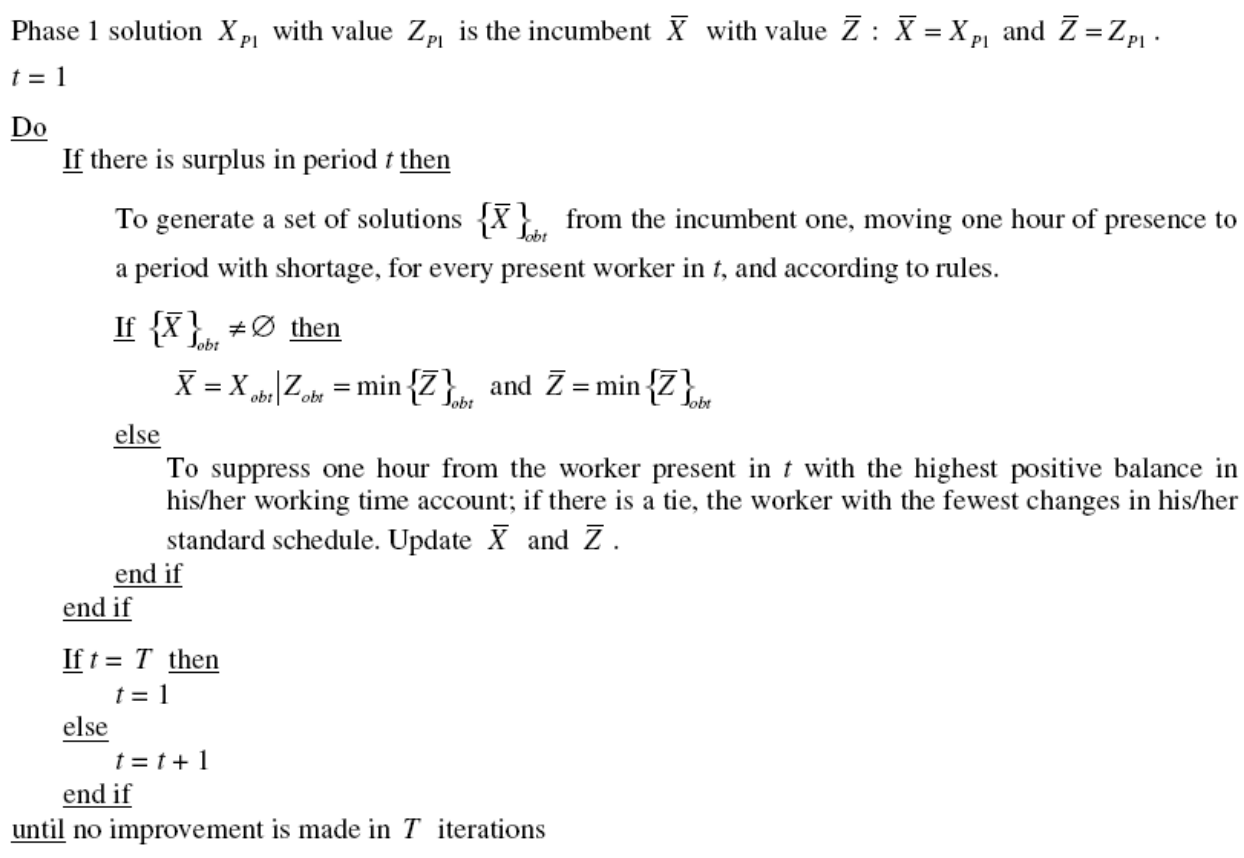

Fig. 4. Pseudo-code of Sub-phase 2.1.

\subsubsection{Sub-phase 2.2: Addition of working hours (shortage case)}

In this final sub-phase, work hours are added to try to eliminate the remaining shortages, if the rules allow this. The solution provided for Sub-phase 2.1 is taken as the initial incumbent solution. Every period of time $t$ is checked to detect shortages. If there is a shortage, one hour of capacity is added to a worker that is not present in $t$, whilst respecting the rules. The worker who has the highest negative balance in his/her work time account is selected, and, in the case of a tie, the worker with the fewest changes in his/her standard schedule is selected. The work hour added is included in the working time account of the worker affected. The process begins in $t=1$ and ends when all periods of the horizon have been considered without any improvement. Fig. 5 shows the pseudo-code of the procedure.

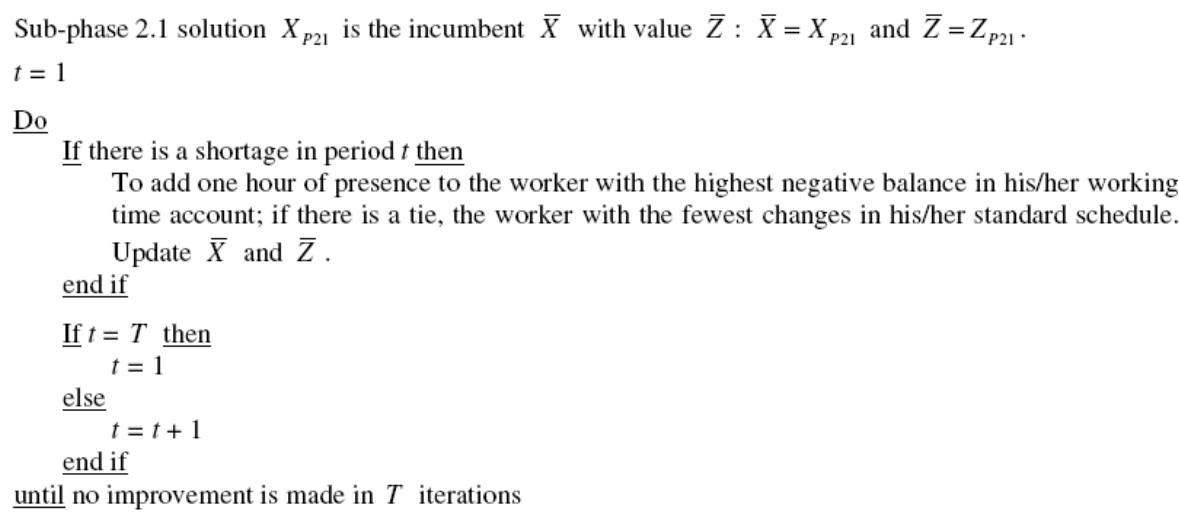

Fig. 5. Pseudo-code of Sub-phase 2.2. 


\subsubsection{Analysis of Phase 2 results}

The time taken to calculate the two sub-phases is very short (less than $30 \mathrm{~s}$ ) and the results obtained are outstanding. The planned capacity is very close to the desired capacity level (see Fig. 6).

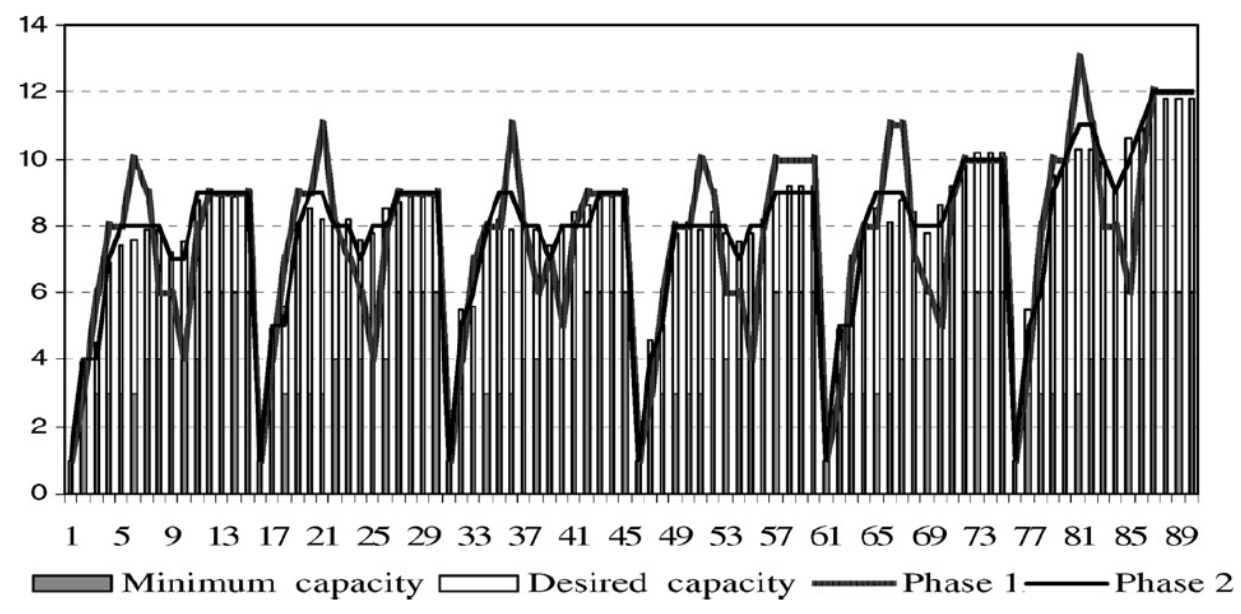

Fig. 6. Example of Phase 2 versus Phase 1.

\section{Procedure validation, results and improvements obtained}

The computational experiment was carried out on a PC Pentium IV $1800 \mathrm{MHz}$ with $256 \mathrm{Mb}$ of RAM. To solve the MILP models, ILOG CPLEX 7.0 optimization software was used. To validate the procedure designed data for two shops with different characteristics were used (here shop T1 and $\mathrm{T} 2$, respectively).

\subsection{Shop T1}

Shop T1 is located on a popular shopping street in the center of a large city. The busiest days are Fridays and Saturdays, but the differences with the other week days are not very marked (see in Fig. 3 an example of the desired and minimum capacity during a week for shop T1). The daily work schedule in shop T1 is from 7 a.m. to 10 p.m., from Monday to Saturday. The planning horizon is divided into 90 one-hour periods and the number of workers is 25. Employees hired to work $40 \mathrm{~h} /$ week can be assigned to 39 different standard schedules, employees who work 30 hours/week can be assigned to 15 standard schedules, employees who work $24 \mathrm{~h} /$ week can be assigned to 50 standard schedules and employees who work $12 \mathrm{~h} /$ week can be assigned to 95 standard schedules. Finally, the minimum and desired capacity levels were also provided.

The first step was a computational experiment to establish the maximum calculation time in which the MILP model can be solved; the test evaluated three options: 120,300 and $900 \mathrm{~s}$. The maximum calculation time was set to $120 \mathrm{~s}$, because the improvement in the results did not counterbalance the longer calculation times. Next, the procedure was applied to 15 weeks. In two of them, the result of the MILP was optimum and in 10 of the 15 weeks, the gap between the solution and the best lower bound was less than $7 \%$. The local optimization only improved 1 of the 13 solutions in which the optimum had not been previously found (however, because of their speed, it was maintained). Finally, Phase 2 was applied.

The results of the new procedure and the solutions obtained manually by the manager of the shop were compared. The comparison is presented in Table 1. The columns show the calculation time in 
seconds (Time); the number of hours of shortage with regard to minimum capacity $\left(T \delta_{M I N}^{-}\right)$; the number of hours of shortage $\left(T \delta^{-}\right)$and surplus $\left(T \delta^{+}\right)$with regard to the desired capacity; and the reduction of work hours obtained by applying the procedure designed with regard to manual planning $(\Delta)$. For the $T \delta_{M I N}^{-}, T \delta^{-}$and $T \delta^{+}$terms, the results obtained by the manual procedure (first) and by the new procedure (second) are provided. They are separated by the symbol "/".

Table 1.

Comparison of results for shop $\mathrm{T} 1$

\begin{tabular}{|c|c|c|c|c|c|}
\hline Week & Time & $T \delta_{M I N}^{-}$ & $T \boldsymbol{\delta}^{-}$ & $T \delta^{+}$ & $\Delta$ \\
\hline 6 & 129 & $0 / 0$ & $3 / 0.3$ & $80.2 / 36.5$ & 41 \\
\hline 7 & 136 & $0 / 0$ & $12.7 / 4.1$ & $47.9 / 28.3$ & 11 \\
\hline 9 & 130 & $0 / 0$ & $1.4 / 0.3$ & $94.9 / 44.8$ & 49 \\
\hline 10 & 152 & $6 / 0$ & $38.3 / 20.6$ & $30.3 / 12.6$ & 0 \\
\hline 11 & 131 & $0 / 0$ & $29.7 / 17.4$ & $16.7 / 4.4$ & 0 \\
\hline 12 & 141 & $0 / 0$ & $15.8 / 2$ & $40.8 / 28$ & 0 \\
\hline 13 & 163 & $0 / 0$ & $50.7 / 15$ & $38.8 / 32.1$ & 29 \\
\hline 14 & 159 & $6 / 0$ & $32.8 / 11.1$ & $57.2 / 58.3$ & 22 \\
\hline 16 & 130 & $2 / 0$ & $11.5 / 1.6$ & $60.4 / 34.5$ & 26 \\
\hline 17 & 131 & $0 / 0$ & $8 / 0$ & $84.9 / 41.9$ & 35 \\
\hline 19 & 138 & $0 / 0$ & $27.9 / 7.4$ & $37.1 / 16.6$ & 0 \\
\hline
\end{tabular}




\begin{tabular}{|l|l|l|l|l|l|}
\hline Week & Time & $T \delta_{\text {MIN }}^{-}$ & $\boldsymbol{T \delta}^{-}$ & $\boldsymbol{T} \boldsymbol{\delta}^{+}$ & $\boldsymbol{\Delta}$ \\
\hline 21 & 149 & $0 / 0$ & $36.6 / 14.9$ & $33.8 / 12.1$ & 0 \\
\hline 22 & 132 & $0 / 0$ & $25.4 / 17.3$ & $41.4 / 17.5$ & 16 \\
\hline 23 & 156 & $8 / 0$ & $59.8 / 43.3$ & $16.8 / 0.3$ & 0 \\
\hline 24 & 144 & $8 / 0$ & $47.9 / 11.9$ & $48.1 / 12.1$ & 0 \\
\hline
\end{tabular}

Table 1 shows that the procedure designed is very efficient. It obtains high quality solutions that in all cases are better that the manual planning procedure (the surplus for Week 14 is only slightly lower in the manual planning). Minimum capacity is reached each week, and shortages and surpluses decrease sharply. Furthermore, remarkable savings in work hours are achieved, and when the saving is nil, using the same number of work hours the shortages and surpluses are always lower. Fig. 7 shows the planned capacity levels obtained manually using the new procedure for Week 24.

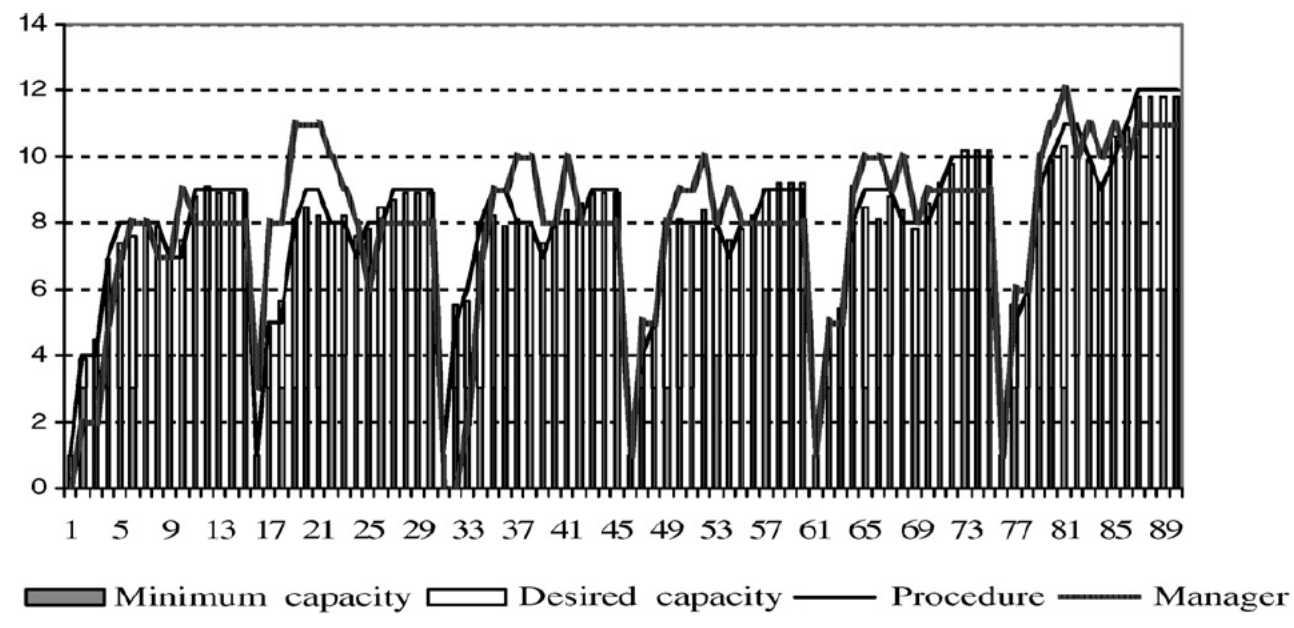

Fig. 7. Example of procedure solution versus manager solution.

\subsection{Shop T2}

Shop T2 is situated in a big mall near a large city and the number of clients increases sharply on Fridays and Saturdays. The daily work schedule is from 7.30 a.m. to 10 p.m., from Mondays to Saturdays, and the planning horizon has 174 half-hour periods. There are 30 workers. Employees who work $40 \mathrm{~h}$ /week can be assigned to 132 different standard schedules, employees who work $30 \mathrm{~h} /$ week can be assigned to 126 standard schedules, employees who work $24 \mathrm{~h} /$ week can be 
assigned to 120 standard schedules and employees who work $12 \mathrm{~h} /$ week workers can be assigned to 220 standard schedules. Finally, the minimum and desired capacity levels were also provided.

The procedure was applied to 8 weeks and the solutions of the new procedure and those obtained manually by the manger were compared, as shown in Table 2. The columns show the time of calculation in seconds (Time); the decrease in the number of hours of shortage with regard to minimum capacity using the procedure designed $\left(\Delta T \delta_{M I N}^{-}\right)$; the decrease in the number of hours of shortage with regard to the desired capacity $\left(\nabla T \delta^{-}\right)$; and the reduction in work hours $(\Delta)$.

Table 2 .

Comparison of results for shop T2

\begin{tabular}{|l|l|l|l|l|}
\hline Week & Time & $\Delta T \delta_{M I N}^{-}$ & $\nabla T \delta^{-}$ & $\Delta$ \\
\hline 38 & 190 & 16 & 52 & 0 \\
\hline 40 & 217 & 9 & 70.8 & 0 \\
\hline 42 & 225 & 25 & 46.6 & 0 \\
\hline 43 & 204 & 10 & 24.9 & 0 \\
\hline 45 & 188 & 0 & 53 & 3 \\
\hline 46 & 180 & 0 & 37.1 & 0 \\
\hline 47 & 168 & 2 & 18.5 & 0 \\
\hline 48 & 179 & 7 & 37.5 & 0 \\
\hline
\end{tabular}

Table 2 shows, again, the speed and efficiency of the procedure designed in comparison to the manual planning carried out by the manager. 


\subsection{Advantages of the procedure}

The set of advantages of the procedure developed are listed below; these improvements were reached jointly by the research team and the company.

- The fulfillment of the minimum capacity requirements has absolute priority.

- The level of service is taken into account as a goal using a non-linear objective function.

- The solutions of the procedure are better than those obtained manually; therefore the new solutions improve the level of service provided to the clients.

- Better use is made of the potential capacity, which decreases overtime.

- There is an absolute fulfillment of the rules agreed concerning the modification of schedules.

- Significant savings are made in the time dedicated to planning the schedules and to managing the data.

- The risk of mistakes has been reduced.

- It is possible to simulate and reschedule.

\section{Conclusions}

This paper presents a procedure for selecting and adapting weekly work schedules with working time accounts in a retail clothing chain. The procedure provides a planned capacity that attempts to guarantee a minimum level of service whenever possible. Furthermore, it takes into account shortages and surpluses with regard to the desired capacity level, using a non-linear objective function: relative shortages and surpluses are minimized and, moreover, they are distributed homogeneously among the different periods.

The procedure designed was validated by the company using data from two shops, one employing 25 workers and the other 30 workers, over a period of several weeks. The solutions of the procedure and those obtained manually by the manger were compared: the procedure designed is very efficient, as it obtains high quality solutions that are in all cases better those obtained by manual planning. Moreover, the new procedure provides other advantages.

Further research could focus on three aspects: to plan a set of shops that share employees; to increase job satisfaction by means of schedules that take into account the employees' personal preferences (by applying these preferences in cases of equality or introducing them in the objective function); and, finally, it is proposed that, due to the fact that the influence of shortages and surpluses in the objective function is non-linear, in each iteration of the algorithms of Sub-phases 2.1 and 2.2, the period with higher shortage or surplus could be considered. 


\section{References}

Berg et al., 2004 P. Berg, E. Appelbaum, T. Bailey and A.L. Kalleberg, Contesting time: International comparisons of employee control of working time, Industrial \& Labor Relations Review 57 (3) (2004), pp. 331-349.

Blochliger, 2004 I. Blochliger, Modelling staff scheduling problems, A tutorial, European Journal of Operational Research 158 (2004), pp. 533-542.

Corominas and Crespán, 1993 Corominas, A., Crespán, J., 1993. Organització del Temps de Treball. Edicions UPC, Barcelona.

Corominas et al., 2005 A. Corominas, J. Ojeda and R. Pastor, Multi-objective allocation of multifunction workers with lower bounded capacity, Journal of the Operational Research Society 56 (6) (2005), pp. 738-743.

Dantzig, 1954 G.B. Dantzig, To comment on Edie's 'traffic delays at toll booths', Operations Research 2 (3) (1954), pp. 339-341.

Day and Ryan, 1997 P.R. Day and D.M. Ryan, Flight attendant rostering for short-haul airline operations, Operations Research 45 (5) (1997), pp. 649-661.

Ernst et al., 2004 A.T. Ernst, H. Jiang, M. Krishnamoorthy, B. Owens and D. Sier, An annotated bibliography of personnel scheduling and rostering, Annals of Operations Research 127 (14) (2004), pp. 21-144.

Freemason et al., 1998 A.J. Freemason, D.M. Ryan and D.M. Panton, Integrated simulation, heuristic and optimisation approaches to staff scheduling, Operations Research 46 (1998), pp. 161-175.

Hung, 1997 R. Hung, Shiftwork scheduling algorithms with phase-delay feature, International Journal of Production Research 35 (7) (1997), pp. 1961-1968.

Keith, 1979 E.G. Keith, Operator scheduling, AIIE Transactions II (I) (1979), pp. 37-41.

Lam et al., 1998 S. Lam, M. Vandenbosch and M. Pearce, Retail sales force scheduling based on store traffic forecasting, Journal of Retailing 74 (1998), pp. 61-88.

Laporte, 1999 G. Laporte, The art and science of designing rotating schedules, Journal of the Operational Research Society 50 (1999), pp. 1011-1017.

Lehndorff, 1999 Lehndorff, S., 1999. New working times systems, work organisation and redistribution of work. Institut Arbeit und Technik Abteilung Arbeitsmarkt, Düsseldorf.

McGinnis et al., 1978 L.F. McGinnis, W.D. Culver and R.H. Deane, One- and two-phase heuristics for workforce scheduling, Computers and Industrialist Engineering 2 (1978), pp. 7-15.

Oke, 2000 Oke, A., 2000. Linking human resource flexibility with manufacturing flexibility: Enablers of labour capacity flexibility in manufacturing plants. In: Proceedings of the First World Conference on Production and Operations Management, Seville, 27 August to 1 September.

Pinker and Larson, 2003 E.J. Pinker and R.C. Larson, Optimizing the use of contingent labour when demand is uncertain, European Journal of Operational Research 144 (1) (2003), pp. $39-55$. 
Sudden and Johns, 1995 M.J. Sudden and T.R. Johns, The effect of demand characteristics on labour scheduling methods, International Journal of Operations and Production Management 15 (1) (1995), pp. 74-88.

Thompson, 1996 G.M. Thompson, A simulated-annealing heuristic for shift scheduling using noncontinuously available employees, Computers and Operations Research 23 (3) (1996), pp. $275-288$.

Thompson, 1997a G.M. Thompson, Assigning telephone operators to shifts at New Brunswick Telephone Company, Interfaces 27 (4) (1997), pp. 1-11.

Thompson, 1997b G.M. Thompson, Work staffing and scheduling models for controlling service levels, Naval Research Logistics 44 (8) (1997), pp. 719-740. 